\title{
Understanding Chemical Reactivity: The Case for Atom, Proton and Methyl Transfers
}

\author{
Luis G. Arnaut* and Sebastião J. Formosinho*[a]
}

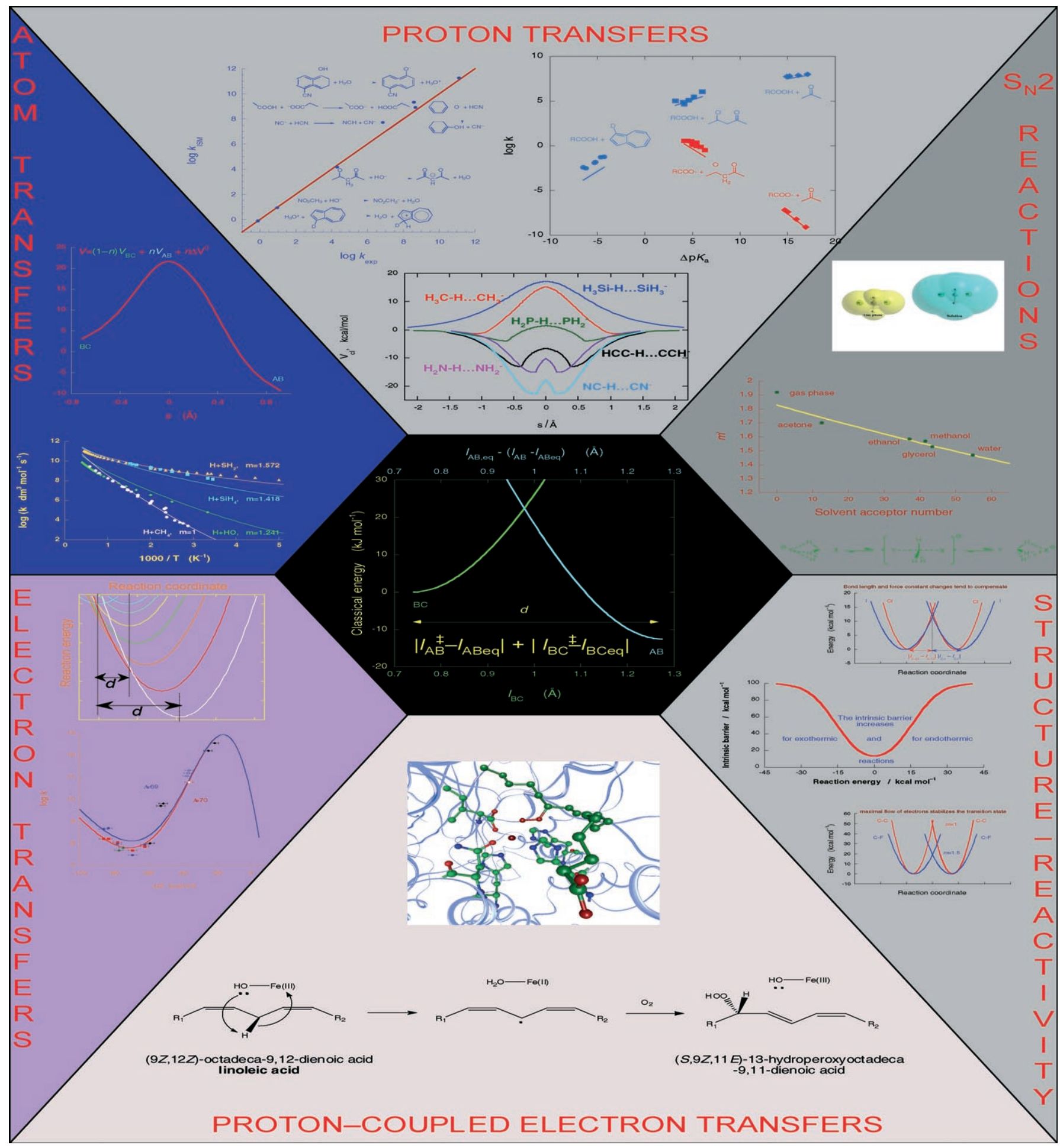


Abstract: The concept of "chemical reactivity" assumes that atoms and molecules contain the necessary information to describe their evolution over time as they transform from reactants to products. This concept was useful in the past to rationalize reactivity trends and predict the behavior of new systems. Free-energy relationships have played a central role in this field. However, electronic effects often counter the energetic effects and give rise to "anomalies" or separate correlations. We discuss a quantification of the concept of "chemical reactivity", emphasizing the role of molecular and electronic factors in Chemistry.

Keywords: atom transfer $\cdot$ nucleophilic substitution • proton transfer • structure-reactivity relationships • transition states

\section{Introduction}

Much of the current understanding of chemical reactivity is based on the hypothesis that the ability of molecules to react rests ultimately on their own structural properties. Although the encounter of two molecules triggers unique features which neither molecule possess alone, notably an electron inflow to the reactive bonds up to a point of saturation, the notion that molecules contain all the information that determines their reactivity has shaped our understanding of chemical reactivity. Conjectures made on the reactivity of new systems depend largely on our ability to relate rate constants with reaction energies and molecular structure in similar systems.

It is one of the fundamental assumptions of our chemical knowledge that analogous substances react similarly and that similar changes in structure produce similar changes in reactivity. While some features are specific for particular reactions others are common to series of reactions, which in some sense constitute a family of reactions. The reactivity changes in an ideal family of reactions are dominated by the changes in a single structural factor. Thus, the underlying approximation is that a family of reactions shares a common broadly defined reaction coordinate, that best represents the changes in the critical structural factor and allows for the establishment of structure-reactivity relationships. In this work we explore such relationships for reactions involving

[a] Prof. Dr. L. G. Arnaut, Prof. Dr. S. J. Formosinho

Chemistry Department, University of Coimbra

3049 Coimbra Codex (Portugal)

Fax: $(+351) 239-827703$

E-mail: lgarnaut@ci.uc.pt sformosinho@qui.uc.pt

Supporting information for this article is available on the WWW under http://www.chemeurj.org/ or from the author. bond-breaking-bond-forming at the transition state, which can be represented as

$\mathrm{A}+\mathrm{BC} \rightarrow \mathrm{AB}+\mathrm{C}$

where the charges have been omitted. The most representative examples of such families of reactions are atom, proton and methyl transfers.

Arrhenius showed in 1889 that a complete theory of reaction rates involves the interpretation of two quantities, a pre-exponential factor, $A$, and the energy of activation, $E_{\mathrm{a}}$. The pre-exponential factor was formulated in 1935 by Eyring $^{[1]}$ in terms of a frequency factor multiplied by a ratio of partition functions, and is conveniently included in the transition-state theory (TST). However, for reactions between neutral species, the pre-exponential factor usually accounts for differences in reactivity of less than three orders of magnitude. In contrast, changes in $E_{\mathrm{a}}$ can easily lead to variations in rates of about 30 orders of magnitude. The calculation of chemically realistic (better than $1 \mathrm{kcal} \mathrm{mol}^{-1}$ ) activation energies has been the cornerstone of chemical reactivity over the last century.

The methods employed to calculate activation energies have relied on numerical solutions of the Schrödinger equation. The remarkable progress of ab initio calculations has made it possible to obtain numerical solutions for a wide range of reactions in the gas phase. This success has overshadowed the valuable results of semiempirical methods, where some information on the reactants and products is employed to estimate activation energies within a family of reactions. In the shift of emphasis from the structure-reactivity relationships of semiempirical methods to the numerical values of $a b$ initio calculations we have tended to lose the physical perception of chemistry as the science that describes matter from the point of view of atomic and molecular properties, and reduce it to electrostatics.

Ideally we would like to calculate accurately the energy barrier of a bond-breaking-bond-forming reaction such as (I), just in terms of the electronic and structural properties of reactants and products, using simple and meaningful relationships between such properties and the energy barrier height. Our contribution to the understanding of chemical reactivity has been focused on the development of a simple but quantitative model that can relate molecular and electronic structure with chemical reactivity using the minimum numerical effort and providing the greatest chemical insight. "Simple" means mathematical tractable such that the "moving parts" of the model can be open to inspection, and calculations can be performed "instantly" for systems of any size. The "chemical insight" means the use of clear concepts, rooted in molecular structure and in electronic properties of the reactive species. In short, we are looking for a model to deconstruct chemical reactivity in its determining factors.

The usefulness of such a model should be measured by its ability to calculate accurate reaction barriers, on one hand, 
and by the rational it provides on the success or failure of widely used physical organic principles, on the other hand. This work first offers a bird's eye view of empirical and semiempirical relationships widely used to interpret atom, proton and methyl transfer rates, and then presents the grounds of a reactivity model that meets the criteria of "simple" and "quantitative". More than focusing on the numerical applications of the model, we emphasises the reactivity trends unveiled by the model and the way they are reflected by structure-reactivity relationships.

\section{Fundamental Structure-Reactivity Relationships}

In the acid-base reaction (II) it appeared reasonable to Brønsted and Pederson ${ }^{[2]}$ that if the rates $k$ at which proton is removed by a particular base $\mathrm{B}^{-}$were compared for various acids HA

$\mathrm{HA}+\mathrm{B}^{-} \rightarrow \mathrm{A}^{-}+\mathrm{BH}$

the base might remove the proton more rapidly from the stronger acids. In fact, relationships between rate of an acid-base reaction and equilibrium have been observed in many cases and frequently obey an equation as the Brønsted catalysis law [Eq. (1)], in which $G_{\mathrm{p}}$ and $\alpha$ are empirical constants, $k$ is the rate for reaction (II) and $K$ the acid dissociation equilibrium constant.

$k=G_{\mathrm{p}} K^{\alpha}$

An equivalent expression can also be obtained for base-catalyzed reactions. Taking into consideration the relationship between rate constants $k$ and $\Delta G^{\neq}$and equilibrium constants $K$ and $\Delta G^{0}$, the equation of Brønsted takes the form of Equation (2), showing that, at a constant temperature, this equation reflects a linear free-energy relationship, LFER. Following a suggestion of Leffler, ${ }^{[3]}$ chemists have tried for a number of years to use the Brønsted coefficient, $\alpha=\partial\left(\Delta G^{\neq}\right) / \partial\left(\Delta G^{0}\right)$, as a measure of the position of the transition state along the reaction coordinate, since usually $0<\alpha<1$, with $\alpha=0$ for reactant-like and $\alpha=1$ for prod-

\footnotetext{
Abstract in Portuguese: $O$ conceito de "Reactividade Química" assume que os átomos e moléculas contêm em si toda a informação necessária para descrever a sua evolução no tempo, quando se transformam de reagentes em produtos. Este conceito foi útil no passado para racionalizar padrões de reactividade e prever o comportamento de novos sistemas. As relações de energia livre tiveram um papel central neste domínio. Porém, os efeitos electrónicos contrariam frequentemente os efeitos energéticos e originam "anomalias" ou correlações independentes. Este trabalho discute uma quantificação do conceito de "Reactividade Química", dando ênfase aos factores moleculares e electrónicos na Química.
}

uct-like transition states.

$\Delta G^{\neq}=\alpha \Delta G^{0}+$ constant

Bell,${ }^{[4]}$ Evans and Polanyi ${ }^{[5]}$ correlated energies of activation, $E_{\mathrm{a}}$, for several reactions in the vapour phase with heats of reaction, $\Delta H^{0}$, according to Equation (3), in which $\alpha_{\mathrm{BEP}}$ and $\Delta E_{0}^{\neq}$are constants. This equation is also related to a LFER. The equation of Bell-Evans-Polanyi (BEP) implies that exothermic reactions will have lower barriers than the endothermic ones. The view that a transition state has structural and energy features that are intermediate between those of starting materials and products is due to Hammond, in $1955,{ }^{[6]}$ who resurrected the view implied by those three authors. The aim of Hammond was that of mechanistic interpretations, postulating that the changes in structure of the TS are affected by the manner in which the substituents affect the energies of intermediates on alternate pathways from reactants to products. Since then this assumption has been known as the Hammond postulate.

$E_{\alpha}=\alpha_{\mathrm{BEP}} \Delta H^{0}+\Delta E_{0}^{\neq}$

Evans and Polanyi have also introduced the concepts of chemical driving force and chemical inertia. ${ }^{[7]} \mathrm{By}$ chemical inertia they meant the work that must be done to produce reaction, partly in breaking a $\mathrm{B}-\mathrm{C}$ bond and in placing the atom A sufficiently close to B. In the present formulation this can be represented by the intrinsic barrier, $\Delta E_{0}^{\neq}$. By chemical driving force they meant the contribution that the energy of formation of the new bond $\mathrm{A}-\mathrm{B}$ makes towards overcoming the inertia, and that is represented by the reaction energy $\Delta E^{0}$, or $\Delta H^{0}$ in Equation (3). Implicit in this treatment is possibility of separating the thermodynamic contribution, $\Delta E^{0}$, and the kinetic contribution, $\Delta E_{0}^{\neq}$, for the energy barrier of the reaction $\Delta E^{\neq}$. A more general formulation was proposed by Marcus in the $1960 \mathrm{~s}^{[8]}$ The equation of Marcus [Eq. (4)] is a quadratic relationship between the barrier of reaction and the reaction free energy. A slightly modified version of Equation (4) was developed for atom and proton transfers. ${ }^{[9]}$ This modification gives the correct limit of $-\Delta E^{\neq}=0$ when $\Delta E^{0}$ approaches infinity, and precludes the onset of an inverted region in atom and proton transfers.

$\Delta G^{\ddagger}=\Delta G_{0}^{\neq}\left(1+\Delta G^{0} / 4 \Delta G_{0}^{\neq}\right)^{2}$

In view of the BEP equation and of the Leffler-Hammond relationship, many chemists were tempted to believe that in a series of related reactions the more reactive species are less selective in their reactivity than less reactive ones. This "reactivity-selectivity principle" (RSP) can be quantified for the relative reactivity of two substances A and B that follow the same type of reaction by two different pathways $\mathrm{X}$ and Y. For example, Figure 1 illustrates the abstraction of primary versus secondary hydrogen atoms of propane by $\mathrm{Cl}$ or 


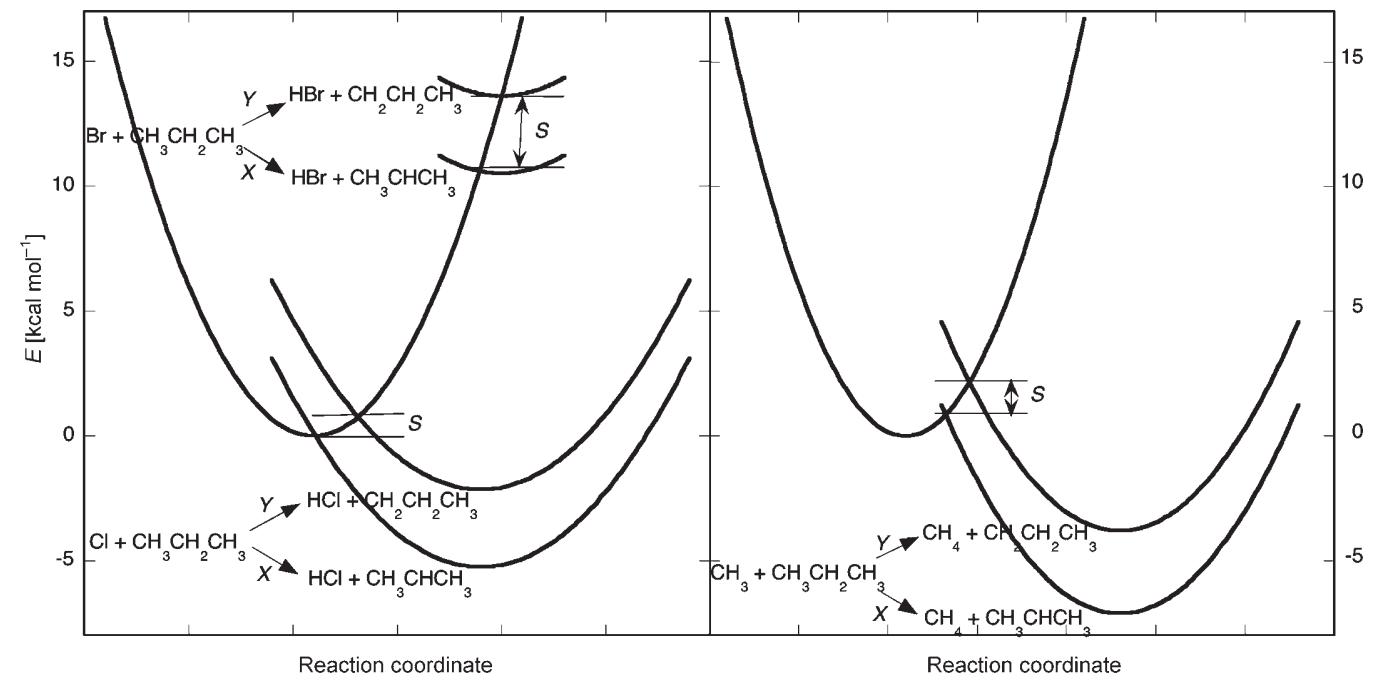

Figure 1. Illustration of the reactivity-selectivity principle for competing primary vs secondary H-atom abstraction reactions $X$ and $Y$ in alkanes.

$\mathrm{Br}$ atoms, which is the classical example of the slowest reaction (abstraction by $\mathrm{Br}$ ) being the most selective. The selectivity $\mathrm{S}$ [Eq. (5)] essentially corresponds to a difference in energy barriers, $\mathrm{S}=\Delta G_{\mathrm{Y}}^{+}-\Delta G_{\mathrm{X}}^{\neq}$. Using the data for the $\mathrm{H}$ atom abstraction from ethane and from a secondary hydrogen of propane, we have $\mathrm{S}(\mathrm{Br})=4.1 \mathrm{kcal} \mathrm{mol}^{-1}$ and $\mathrm{S}(\mathrm{Cl})=$ $0.2 \mathrm{kcalmol}^{-1}$. $^{[10,11]}$ Although such examples support RSP, Mayr pointed out that they are biased by the nearly barrierless nature of the most reactive reactions. ${ }^{[12]}$ The criticism of Mayr is entirely justified, but it should be tempered by examples such as the abstraction of primary vs. secondary $\mathrm{H}$ atoms of alkanes by the methyl group, which exhibit intermediate reactivities and selectivities when compared with $\mathrm{Cl}$ and $\mathrm{Br}$ abstractions, and are not barrierless. Using the abstractions from ethane and propane as previously, the selectivity is $\mathrm{S}\left(\mathrm{CH}_{3}\right)=2.2 \mathrm{kcal} \mathrm{mol}^{-1}$. ${ }^{[13]}$

$\mathrm{S}=\log \left(k_{\mathrm{X}} / k_{\mathrm{Y}}\right)$

Free-energy relationships suggest that reactions with strong driving forces thermodynamically will also proceed rapidly. However, such an implication does not hold generally. For elementary reactions, the correct interpretation of reactivity rests on the values of the intrinsic barriers and their relation to molecular and electronic structure.

In 1937 Hammett realized that the addition of a substituent in the aromatic ring has a quantitative effect on the dissociation constant $K$, and proposed a relationship to account for such findings ${ }^{[14]}$ [Eq. (6)]. The same kind of expression is also valid for the rate constants, $k$, and can be expressed in terms of free energies of activation [Eq. (7)], in which $K^{0}$ and $\Delta G_{0}^{\neq}$correspond to the values of the "parent" or "unsubstituted" compound. The substituent constant $\sigma$ is a measure of the electronic effect of replacing $\mathrm{H}$ by a given substituent (in the para or meta position) and is, in principle, independent of the nature of reaction. The reaction constant $\rho$ depends on the nature of the reaction, and is a measure of the susceptibility of the reaction to the electronic effects of substituents. Other scales of substituent effects, such as that of Taft $\left(\sigma^{*}\right)$, were developed for aliphatic compounds.

$\log K=\log K^{0}+\rho \sigma$

$\Delta G^{\neq}=\Delta G_{0}^{\neq}-2.3 R T \rho \sigma$

Equation (6) raises the issue of how electronic effects contribute to the making of reaction barriers. Chemical reactions often involve some charge transfer between reactant molecules (or between two different parts of one molecule), and several reactivity indexes express electronic effects. A particularly fruitful index is the chemical hardness, defined as one-half of the second derivative of the ground-state electronic energy $E$ with respect to $N$ electrons of the system, ${ }^{[15,16]}$ or, using the finite difference approximation, defined by Equation (8), in which $I_{\mathrm{P}}$ is the ionization potential and $E_{\mathrm{A}}$ is the electronic affinity. The curvature of $E$ versus $N$ plots reflects the resistance of the system to change its number of electrons. The inverse of the hardness is the softness, $\sigma=1 / \eta$. According to Koopmans's theorem, the hardness of a spin-paired molecule is half the energy gap between the HOMO and the LUMO. For a two-partner electron transfer reaction, the hardness of the initial system is half the energy difference between the HOMO of the electron donor and the LUMO of the electron acceptor. ${ }^{[16]}$ Based on circumstantial evidence and variational principles, Pearson and Parr proposed a maximum hardness principle according to which chemical systems tend to become as hard as possible, or, stated differently, soft molecules are more reactive than hard molecules. ${ }^{[17-19]}$

$\eta=\left(I_{\mathrm{p}}-E_{\mathrm{A}}\right) / 2$

According to the maximum hardness principle, it should be possible to relate the barrier height $\Delta E^{\ddagger}$ to the hardness $\eta$. 
Shaik and Pross followed an independent approach based on valence bond (VB) mixing diagrams to arrive at a similar concept for processes which involve combinations of electrophile and nucleophile, or donor and acceptor. ${ }^{[2-22]}$ In the $\mathrm{VB}$ approach the barrier height is express as a fraction $f$ of the energy gap $g$ between the reactants ground state and the vertical excited state that possesses the electron pairing scheme of the product, as shown by Equation (9), in which $B$ is the quantum mechanical resonance energy of the transition state. In proton transfers, hydride transfers, $\mathrm{S}_{\mathrm{N}} 2$ reactions, cation-anion recombinations or electron transfer reactions, $g$ is expressed as the difference between the $I_{\mathrm{P}}$ of one reactant and the $E_{\mathrm{A}}$ of the other, that is, as the vertical charge-transfer energy. For example, the promotion energy in $\mathrm{S}_{\mathrm{N}} 2$ reactions is $g=I_{\mathrm{P}}(\mathrm{N}:)-E_{\mathrm{A}}(\mathrm{RX})=2 \eta$, where $I_{\mathrm{P}}(\mathrm{N}:)$ is the ionization potential of the nucleophile and $E_{\mathrm{A}}(\mathrm{RX})$ is the electron affinity of the substrate. ${ }^{[20]}$

$\Delta E^{\neq}=f g-B$

It must be emphasized that neither Shaik nor Pearson and Parr scaled chemical reactivity exclusively to charge-transfer interactions. For example, the energy gap of VB diagrams corresponding to bond-breaking-bond-forming reactions of predominantly covalent bonds, such as atom abstraction reactions, is given by the singlet-triplet excitation energy of the reactive bond. In addition to the reactivity indexes that measure resistance to change in the electron density of a molecule, Pearson gauged the resistance to change in the nuclear position by the molecular hardness, ${ }^{[23]}[$ Eq. (10)], in which $f$ is the force constant of the bond

molecular hardness $=f\left(l_{\mathrm{eq}}\right)^{2}$

The expression of the relative importance of covalent and charge-transfer interactions, and the separability of intrinsic and thermodynamic contributions to the reaction barrier, delayed the emergence of a simple reactivity model of broad scope. Our endeavour to discover theoretical principles for understanding chemical reactivity led us to a model that integrates all these factors, called Intersecting/Interacting State Model.

\section{Intersecting/Interacting-State Model}

The Intersecting/Interacting State Model (ISM) is based on three fundamental assumptions. The first was borrowed from the BEBO model of Johnston and Parr, ${ }^{[24]}$ and states that "...the bond order is preserved along the reaction coordinate...", as expressed by Equation (11) in which $n_{\mathrm{AB}}$ is the bond order of the new bond formed in the products bond and $n_{\mathrm{BC}}$ is the bond order of the bond that is broken in the reactants. There are several fundamental studies that support this approximation. ${ }^{[25-27]}$ Intuitively, we would expect that, for a symmetrical reaction (reaction energy $\Delta V^{0}=0$ ), the synchronization of the two events would give a transition state bond order $n^{\neq}=0.5$. When the energy of new bond $\left(V_{\mathrm{AB}}\right)$ and that of the old bond $\left(V_{\mathrm{BC}}\right)$ are expressed in terms of the reaction coordinate $n$, the energy of the transition state is just $V_{\mathrm{BC}}\left(n^{\neq}=0.5\right)=V_{\mathrm{AB}}\left(n^{\neq}=0.5\right)$ for a symmetrical reaction, or more generally $V_{\mathrm{BC}}(n)=V_{\mathrm{AB}}(n)+\Delta V^{0}$. The practical use of this formulation depends on the relation between transition-state bond orders and bond extensions, since the latter are more easily related to the energy of the system.

$n=n_{\mathrm{AB}}=1-n_{\mathrm{BC}}$

The second assumption of ISM builds on the Pauling relation between bond lengths and bond orders. ${ }^{[28]}$ This relation is generalized to transition states in Equation (12), where $a_{\text {sc }}^{\prime}$ is a "universal" constant. ISM relates transition state bond lengths $\left(l^{\neq}\right)$to the corresponding bond orders $\left(n^{*}\right)$ and to the equilibrium bond lengths of reactants and products $\left(l_{\mathrm{BC}, \mathrm{eq}}\right.$ and $\left.l_{\mathrm{AB}, \mathrm{eq}}\right)$. The bond extension is scaled by the sum of the equilibrium bond lengths of reactant and product, because the transition state has two bonds and a long bond will extend more than a short one. The value of $a_{\mathrm{sc}}^{\prime}$ can be obtained from the most exact potential energy surface of a bond-breaking-bond-forming reaction, which is the $\mathrm{H}+\mathrm{H}_{2}$ reaction. For this symmetrical reaction $\left(n^{\neq}=0.5\right)$, the ab initio $l^{\neq}$is reproduced with $a_{\mathrm{sc}}^{\prime}=0.182 .{ }^{[29]}$ For exothermic reactions it was possible to show that the sum of the bond extensions, $d$, takes approximately the form of Equation (13) in which $\Omega$ is a constant for a family of reactions. ${ }^{[30,31]}$ The Frontispiece illustrates the role of $d$. According to Equation (13), the reduced bond lengths, $\delta=d /$ $\left(l_{\mathrm{BC}, \mathrm{eq}}+l_{\mathrm{AB}, \mathrm{eq}}\right)$, of isothermic reactions should be 0.25 . The data in the Supporting Information show that the average reduced bond length of eight $\mathrm{H}$-atom abstraction reactions with $\left|\Delta V^{0}\right|<3 \mathrm{kcalmol}^{-1}$ is $\delta=0.24$, with a standard deviation of 0.02. Equation (13) also predicts an increase of $d$ with $\left(\Delta V^{0}\right)^{2}$. It is difficult to find a series of closely related reactants that vary substantially in $\Delta V^{0}$ and have reliable transition-state bond lengths, to test this equation. However, the $\mathrm{H}$-atom transfers represented in Figure 2 show that the reduced bond lengths approximately follow the quadratic relationship of Equation (13) even though they do not qualify as "one" family of reactions.

$$
\begin{aligned}
& l_{\mathrm{AB}}^{ \pm}-l_{\mathrm{AB}, \text { eq }}=-a_{\mathrm{sc}}^{\prime}\left(l_{\mathrm{AB}, \mathrm{eq}}+l_{\mathrm{BC}, \mathrm{eq}}\right) \ln \left(n^{\mp}\right) \\
& l_{\mathrm{BC}}^{+}-l_{\mathrm{BC}, \mathrm{eq}}=-a_{\mathrm{sc}}^{\prime}\left(l_{\mathrm{AB}, \mathrm{eq}}+l_{\mathrm{BC}, \mathrm{eq}}\right) \ln \left(1-n^{\neq}\right) \\
& d=\left|l_{\mathrm{AB}}^{ \pm}-l_{\mathrm{AB}, \mathrm{eq}}\right|+\left|l_{\mathrm{BC}}^{\neq}-l_{\mathrm{BC}, \mathrm{eq}}\right| \\
& =2 a_{\mathrm{sc}}^{\prime}\left(l_{\mathrm{AB}, \mathrm{eq}}+l_{\mathrm{BC}, \mathrm{eq}}\right)\left[\ln (2)+\left(\Delta V^{0} / 2 \Omega\right)^{2}\right]
\end{aligned}
$$

The reasonable agreement between the bond extensions of ISM and ab initio methods, make Equation (12) attractive to calculate transition state structures. From such structures and the (harmonic or Morse) functions relating the potential 


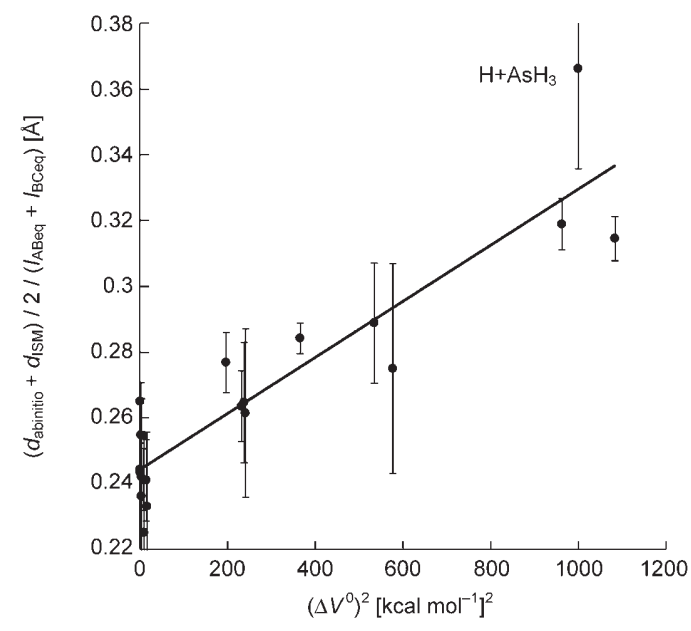

Figure 2. Relation between the reduced semi-sum of ISM and ab initio transition-state bond extensions, and the square of the reaction energy of $\mathrm{H}$-atom transfers. The error bars are a measure of the difference between the two methods. The ab initio transition-state bond lengths of the $\mathrm{H}+$ $\mathrm{AsH}_{3}$ hydrogen abstraction seem to be overestimated. The data and references to the literature are available in Supporting Information.

energies of $\mathrm{BC}$ and $\mathrm{AB}$ to the respective bond lengths, it is possible to calculate the energy of the transition state. However, before pursuing this route, we need to consider the possibility of an additional interaction between $\mathrm{A}, \mathrm{B}$ and $\mathrm{C}$ at the transition state to formulate the last assumption of ISM.

The last assumption concerns the method to account for the electronic stabilization when $\mathrm{A}$ and $\mathrm{B}-\mathrm{C}$ interact at the

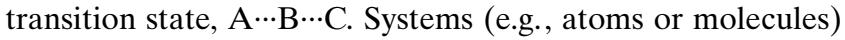
coming together must attain a common electronic chemical potential at equilibrium. The chemical potential $\left(\mu_{\mathrm{el}}\right)$ is the partial derivative of the ground-state electronic energy $E$ with respect to $N$ electrons of the system. It is identical to the negative of the electronegativity $\left(\chi_{M}\right)$, as defined by Mulliken, when a finite-difference approximation is employed to express the chemical potential, $\mu_{\mathrm{el}}=-\left(I_{\mathrm{P}}+E_{\mathrm{A}}\right) /$ $2{ }^{[15]}$ The chemical potential, being synonymous with electronegativity, characterizes the tendency of electrons to escape from the equilibrium system. When $\mathrm{A}$ and $\mathrm{B}-\mathrm{C}$ are brought together, electrons will flow from the species of lower $\chi_{\mathrm{M}}$ to that of higher $\chi_{\mathrm{M}}$, following the principle of electronegativity equalization. Whereas the electronegativities drive the electron transfer, hardness acts as a resistance. Thus, we may express the lowering of the total binding energy of the transition state in terms of the maximal flow of electrons from $\mathrm{A}$ or from $\mathrm{C}$ which saturates $\mathrm{A} \cdots \mathrm{B} \cdots \mathrm{C}$. Parr has shown that, within the framework of a valence-state parabola model, this is given by ${ }^{[32]}$ Equation (14) in which we introduce the variable $m$ for consistency with our earlier nomenclature.

$\Delta N_{\max }=-\mu_{\mathrm{el}} / \eta_{\mathrm{el}}=\left(I_{\mathrm{p}}+E_{\mathrm{A}}\right) /\left(I_{\mathrm{p}}-E_{\mathrm{A}}\right)=m$

The energy lowering due to partial electron transfer is only a part of the energy change associated with the formation of the transition state.$^{[33]}$ A pervasive contribution is due to the covalent bonding interaction present in $\mathrm{A} \cdots \mathrm{B} \cdots \mathrm{C}$, but this has already been taken into account by the conservation of the bond order. Clearly, the electrophilicity index $m$ is a measure of the extra electronic stabilization of the transition state. It increases with the propensity of the "ligands" A and $\mathrm{C}$ to participate in partial electron transfer with the transition state (low $I_{\mathrm{P}}$ and/or high $E_{\mathrm{A}}$ ), but otherwise leads to no stabilization (high $I_{\mathrm{P}}$ and/or low $E_{\mathrm{A}}$ ). The barrier of a chemical reaction is due to the chemical hardness (resistance to change in the electron distribution) and to molecular hardness (resistance to change in the nuclear positions). ${ }^{[18]} \mathrm{We}$ formulate the last assumption of ISM expressing the molecular and chemical hardnesses together in the making of the reaction barrier, and tempering the latter by the chemical potential, as shown in Equations (15), in which the reactive bonds are modelled by harmonic oscillators. This, with Equation (12), suffices to calculate barrier heights when the equilibrium bond lengths, force constants, ionization potentials and electronic affinities of reactants and products are known.

$$
\begin{aligned}
& V_{\mathrm{BC}}^{\neq}=0.5 f_{\mathrm{BC}} / m^{2}\left(l_{\mathrm{BC}}^{\neq}-l_{\mathrm{BC}, \mathrm{eq}}\right)^{2} \\
& V_{\mathrm{AB}}^{\neq}=0.5 f_{\mathrm{AB}} / m^{2}\left(l_{\mathrm{AB}}^{ \pm}-l_{\mathrm{AB}, \mathrm{eq}}\right)^{2}
\end{aligned}
$$

The transfer of hydrogen, proton or hydride in any of their isotopic forms is subject to significant tunnelling corrections, which can only be calculated when the whole reaction energy profile is known. For such cases, it is necessary to make an interpolation between the classical potentialenergy curves of reactant $\mathrm{B}-\mathrm{C}$ bond and product $\mathrm{A}-\mathrm{B}$ bond. The simplest, linear interpolation along the reaction coordinate expressed in terms of the bond order coordinate is given by Equation (16), in which $\Delta V^{0}$ is the classical energy of the reaction $\left(\Delta V^{0}=D_{\mathrm{e}, \mathrm{BC}}-D_{\mathrm{e}, \mathrm{AB}}\right)$, and the potentials $V_{\mathrm{BC}}$ and $V_{\mathrm{AB}}$ are conveniently represented by Morse potentials, $V=D_{\mathrm{e}}\left\{1-\exp \left[-\beta\left(l-l_{\text {eq }}\right)\right]\right\}^{2}$, where the bond extensions take the form of Equation (12). From the relation between the force constant, the electronic dissociation energy and the spectroscopic constant of a bond, $f=2 D_{\mathrm{e}} \beta^{2}$, the inclusion of the electrophilicity index in the Morse curve corresponds to the replacement of $\beta$ by $\beta / m$. The reaction coordinate can be further sophisticated to include zero-point energy corrections and the change of $m$ from unity in the reactants, to the value given by Equation (14) at the transition state, and back to unity in the products. ${ }^{[34]}$

$V_{\mathrm{el}}=(1-n) V_{\mathrm{BC}}+n V_{\mathrm{AB}}+n \Delta V^{0}$

One of the most important perturbations in the reaction coordinate of Equation (16) is the occurrence of hydrogen bonding. When $\mathrm{B}$ is a hydrogen atom or a proton and the transfer occurs between electronegative atoms, two complexes separated by an internal barrier are present in the reaction coordinate. The energy, bond lengths and frequency 
of a H-bond are related by the Lippincott-Schroeder potential. ${ }^{[35]}$ The full description of the energy profile now requires information on at least one of these properties. With one of them, the other two can then be calculated, as well as the bond order of the $\mathrm{H}$-bond. The presence of an $\mathrm{H}$ bond in the ISM reaction coordinate is regarded an incipient hydrogen (or proton) transfer, with a $\mathrm{H} \cdots \mathrm{A}$ bond order greater than zero, that is, the reaction coordinate is advanced with respect to the isolated reactants, and the reaction barrier may be reduced. ${ }^{[36]}$

\section{Barrier Heights, Rate Constants and Activation Energies}

Using the three assumptions of ISM, scaling of the bond lengths with $a_{\mathrm{sc}}^{\prime}=0.182$ and using the Lippincott-Schoeder potential for H-bonded systems, we have calculated the classical barrier heights of 51 systems. Figure 3 compares the barrier heights of $\mathrm{H}$-atom and proton transfers calculated by high-level ab initio methods and by ISM, together with the recently published barriers heights of representative methyl transfers.

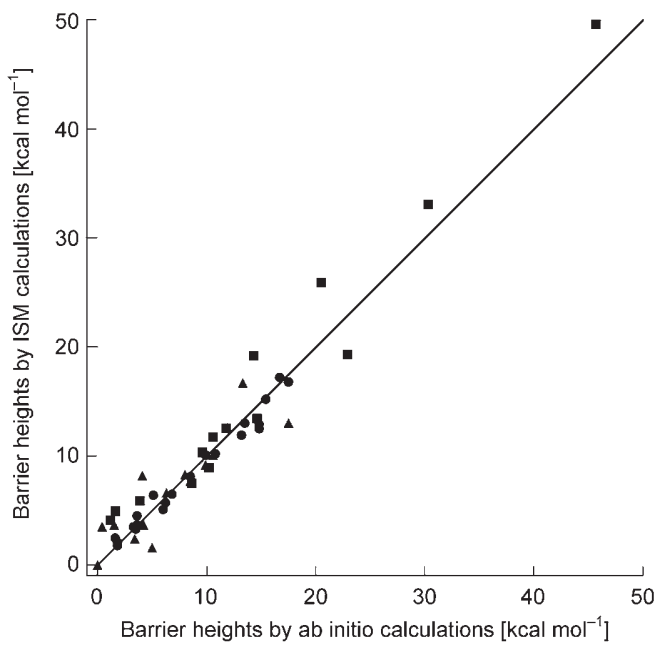

Figure 3. Correlation between barriers heights calculated by ISM and ab initio methods (correlation coefficient: 0.973). • classical barriers of $\mathrm{H}$ atom transfers $;^{[37]} \boldsymbol{\Lambda}$ : barriers heights of $\mathrm{H}$-atom and proton transfers in H-bonded systems $;^{[36]}$ a: classical barriers of methyl transfers $\left(1 \mathrm{kcalmol}^{-1}\right.$ was added to the $\mathrm{G} 2$ barrier to compare with the classical barrier of ISM). ${ }^{[38]}$ The line is the ideal correlation. Data and references to the literature are available as Supporting Information.

The ultimate goal of any reactivity model is to predict the rate constant, $k(T)$, of an elementary reaction in any reaction conditions with the level of accuracy of experimental techniques. Equation (16) allows one to estimate the classical reaction energy profile along the whole reaction coordinate. Zero-point energy effects can also be incorporated in the reaction energy profile in order to estimate a vibrationally adiabatic barrier, $\Delta V_{\text {ad }}^{\neq}$Stretching and bending vibrational frequencies of the transition state are estimated from the Wilson equation for the symmetric and antisymmetric stretching frequencies for a linear triatomic system; the antisymmetric stretching is transformed in the reaction coordinate and an empirical correlation between stretching and bending frequencies in triatomic molecules is employed to estimate the bending frequencies. Figure 4 compares the rates calculated for nearly $100 \mathrm{H}$-atom abstractions, proton transfers and methyl transfers with the experimental data, both in the gas phase and solution. TST is employed for such calculations, which incorporate tunnelling corrections, solvent effects and specific hydrogen bonding using the Lippincott-Schroeder potential.

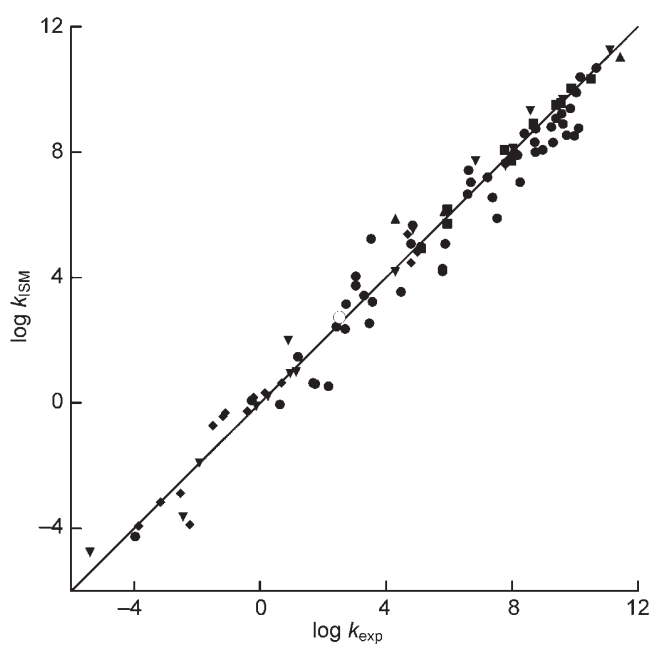

Figure 4. Correlation between experimental and ISM calculated rates of $\mathrm{H}$-atom, proton and methyl transfers (correlation coefficient: 0.987). •: $\mathrm{H}$-atom abstractions from Table 4 of ref. [37]; a: H-atom abstractions in H-bonded systems from ref. [36]; $\mathbf{\Delta}$ : intramolecular $\mathrm{H}$-atom and proton transfers from ref. [39] and proton transfers from Table 3 of ref. [34] lozenges: methyl transfers from Figure 6 of ref. [38]; $\circ$ : enzyme catalyzed $\mathrm{H}$-atom transfers from ref. [40]. The line refers to the ideal correlation.

The activation energy is a phenomenological quantity, related to the internal energy of activation, which, in turn, can be related to classical barrier height $\left(\Delta V^{\ddagger}\right)$, to the difference in zero-point energies of transition state and reactants, and to the difference in internal energies between the transition state and the reactants. The comparison between $E_{\mathrm{a}}$ and barrier heights is further complicated by the presence of tunnelling, which increases the temperature dependence of the observed $E_{\mathrm{a}}$. All these effects were taken into account in the calculation of the reaction rates presented in Figure 4, although for the purpose of a simple calculation of activation energies, it is more convenient to re-scale ISM to the activation energy of the $\mathrm{H}+\mathrm{H}_{2}$ reaction, rather than to its transition-state geometry, and use the new scaling to calculate the activation energies of other $\mathrm{H}$-atom transfers. The scaling to $E_{\mathrm{a}}=7.5 \mathrm{kcal} \mathrm{mol}^{-1}$ given by the relative rates measured by Quickert and Le Roy ${ }^{[41]}$ converted to absolute rate constants using the expression of Michael,,$^{[42]}$ gives $a^{\prime}=0.156 .{ }^{[43]}$ With this scaling it is very simple to calculate other activation energies. Figure 5 shows the comparison between experimental 


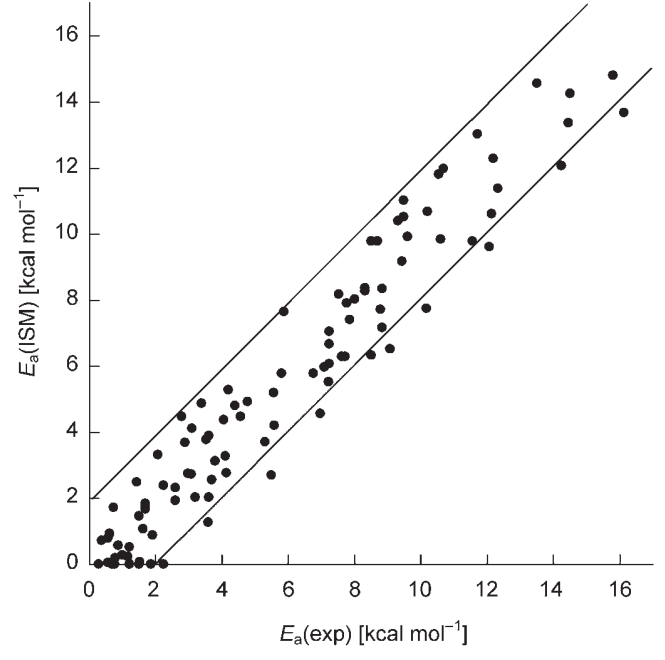

Figure 5. Experimental and calculated activation energies of $\mathrm{H}$-abstraction reactions and proton transfers, from ref. [37].

and calculated activation energies of $100 \mathrm{H}$-atom transfers. The correlation coefficient is 0.965 and the average error is less than $1 \mathrm{kcal} \mathrm{mol}^{-1}$, much better than may initially be anticipated for such a simple model. Again, the comparison with the experiment shows that ISM takes into account the parameters that control the reactivity of this class of reactions.

\section{A Rational for Structure-Reactivity Relationship}

The transition-state structures and energies of "small" systems in Figures 2 and 3 calculated with ISM are in excellent agreement with those of the best available ab initio calculations. The agreement between calculated and experimental rates for a very wide range of reactions in Figures 4 and 5 is impressive and unprecedented for absolute rate calculations. However, we prefer to emphasize the new physical insights provided by ISM, which relate molecular structure to chemical reactivity.
ISM organizes the structures of the transition state following two previously unsuspected guidelines: the transitionstate bond extensions are proportional to the equilibrium bond lengths and their sum tends to increase with the exothermicity of the reactions. Indeed, as the reactions become more exothermic, $n^{\neq}$tends to zero and the product bond extension tends to infinity. This corollary, exposed in Figure 2, has profound consequences in structure-reactivity relationships, because it predicts from a strictly theoretical point of view that "intrinsic" barriers tend to increase with the exothermicity of the reactions.

In Table 1 we assembled sets of systems where, other reactivity factors being almost equal, the variation of one factor dominates. Let us first consider thermoneutral $\mathrm{H}$-abstractions such as $\mathrm{H}+\mathrm{H}_{2}$ and $\mathrm{CH}_{3}+\mathrm{CH}_{4}$. Table 1 reveals that the only relevant difference between the two systems is the sum of the equilibrium bond lengths: in the second system this structural factor is about $47 \%$ larger and the energy barrier is $66 \%$ higher. Other structural factors, such as the pyramidization of the carbon atom, can be excluded because there is not a systematic difference between ISM and ab initio barriers heights in the series: $\mathrm{H}+\mathrm{H}_{2} \mathrm{H}+\mathrm{CH}_{4}$, $\mathrm{CH}_{3}+\mathrm{CH}_{4}$ and $\mathrm{CH}_{3} \mathrm{CH}_{2}+\mathrm{CH}_{3} \mathrm{CH}_{3}$. Thus shorter bonds can act as a kind of driving force for chemical reactions; this is particularly relevant for reactions where $\mathrm{H}_{2}$ is involved. The same effect is also observed in the reaction $\mathrm{H}+\mathrm{HCl}$ and $\mathrm{Cl}+\mathrm{CH}_{4}$, which have reaction energies of -1.0 and $+1.7 \mathrm{kcal} \mathrm{mol}^{-1}$, respectively. However, the barriers of the reactions involving hydrogen transfer to chorine are much smaller than those involving hydrogen transfer to carbon. In this case, the major difference is in the electronic $m$ parameter. The reactions with the higher $m$ values have lower energy barriers. This reveals that the electrophilicity index also has a profound effect on intrinsic barriers. The methyl group transfers of the type $\mathrm{X}^{-}+\mathrm{CH}_{3} \mathrm{X}$ provide a paradigmatic example of another reactivity factor. Along the series $\mathrm{X}=\mathrm{Cl}, \mathrm{Br}$, I, the value of $m$ is approximately constant while $l_{\mathrm{AB}, \mathrm{eq}}$ increases and the force constant of the $\mathrm{C}-\mathrm{X}$ bond decreases. The compensation between the increase in the bond length and the increase of the force constant keeps the reaction barriers approximately constant along this group of the

Table 1. Effect of structural and electronic parameters on the energy barrier of thermoneutral or nearly thermoneutral reactions.

\begin{tabular}{|c|c|c|c|c|c|c|}
\hline \multirow[t]{2}{*}{ Reaction } & \multirow[t]{2}{*}{$l_{\mathrm{AB}, \mathrm{eq}}+l_{B \mathrm{C}, \mathrm{eq}}[\AA]$} & \multirow[t]{2}{*}{$D_{\mathrm{e}}\left[\mathrm{kcal} \mathrm{mol}^{-1}\right]$} & \multirow[t]{2}{*}{$\beta\left[\AA^{-1}\right]$} & \multirow[t]{2}{*}{$m$} & \multicolumn{2}{|c|}{$\Delta V_{\mathrm{cl}}^{\neq}\left[\mathrm{kcal} \mathrm{mol}^{-1}\right]$} \\
\hline & & & & & ISM & $\mathrm{ab}$ initio \\
\hline $\mathrm{H}+\mathrm{H}_{2} \rightarrow \mathrm{H}_{2}+\mathrm{H}$ & 1.483 & 104.2 & 1.935 & 1 & 10.1 & $9.9^{[\mathrm{a}]}$ \\
\hline $\mathrm{H}+\mathrm{CH}_{4} \rightarrow \mathrm{H}_{2}+\mathrm{CH}_{3}^{\cdot}$ & 1.828 & & & 1 & 12.9 & $14.7^{[\mathrm{b}]}$ \\
\hline $\mathrm{CH}_{3}^{\circ}+\mathrm{CH}_{4} \rightarrow \mathrm{CH}_{4}+\mathrm{CH}_{3}^{\circ}$ & 2.174 & 104.9 & 1.823 & 1 & 16.8 & $17.5^{[\mathrm{c}]}$ \\
\hline $\mathrm{C}_{2} \mathrm{H}_{5}{ }^{\circ}+\mathrm{C}_{2} \mathrm{H}_{6} \rightarrow \mathrm{C}_{2} \mathrm{H}_{6}+\mathrm{C}_{2} \mathrm{H}_{5} \cdot$ & 2.188 & 101.1 & 1.883 & 1 & 17.2 & $16.7^{[\mathrm{c}]}$ \\
\hline $\mathrm{H}+\mathrm{HCl} \rightarrow \mathrm{H}_{2}+\mathrm{Cl}$ & 2.016 & 103.2 & 1.867 & 1.773 & 5.1 & $5.7^{[\mathrm{c}]}$ \\
\hline $\mathrm{Cl}+\mathrm{CH}_{4} \rightarrow \mathrm{HCl}+\mathrm{CH}_{3}$ & 2.362 & 103.2 & 1.823 & 1.773 & 6.5 & $7.6^{[\mathrm{d}]}$ \\
\hline $\mathrm{Br}^{-}+\mathrm{CH}_{3} \mathrm{Br} \rightarrow \mathrm{BrCH}_{3}+\mathrm{Br}^{-}$ & 3.866 & 72.1 & 1.537 & 1.796 & 12.6 & \\
\hline $\mathrm{I}^{-}+\mathrm{CH}_{3} \mathrm{I} \rightarrow \mathrm{ICH}_{3}+\mathrm{I}^{-}$ & 4.264 & 57.6 & 1.536 & 1.828 & 11.7 & \\
\hline $\mathrm{CH}_{3} \mathrm{CH}_{2}^{-}+\mathrm{CH}_{3} \mathrm{CH}_{2} \mathrm{CH}_{3} \rightarrow \mathrm{CH}_{3} \mathrm{CH}_{2} \mathrm{CH}_{3}+\mathrm{CH}_{3} \mathrm{CH}_{2}^{-}$ & 3.064 & 88.5 & 1.811 & 0.938 & 49.6 & \\
\hline $\mathrm{CH}_{3} \mathrm{NH}^{-}+\mathrm{CH}_{3} \mathrm{NHCH}_{3} \rightarrow \mathrm{CH}_{3} \mathrm{NHCH}_{3}+\mathrm{CH}_{3} \mathrm{NH}^{-}$ & 2.910 & 82.2 & 1.999 & 1.161 & 33.1 & \\
\hline $\mathrm{CH}_{3} \mathrm{O}^{-}+\mathrm{CH}_{3} \mathrm{OCH}_{3} \rightarrow \mathrm{CH}_{3} \mathrm{OCH}_{3}+\mathrm{CH}_{3} \mathrm{O}^{-}$ & 2.832 & 82.9 & 2.094 & 2.094 & 25.9 & \\
\hline
\end{tabular}

[a] Ref. [44]. [b] Ref. [45]. [c] Ref. [46]. [d] Ref. [47]. 
Periodic Table. The same does not happen along the period $\mathrm{X}=\mathrm{N}, \mathrm{C}, \mathrm{O}, \mathrm{F}$ and the reactivity is again dominated by the increase in $m$. Figure 6 illustrates the effect of these reactivity parameters on the barrier height using harmonic oscillators.

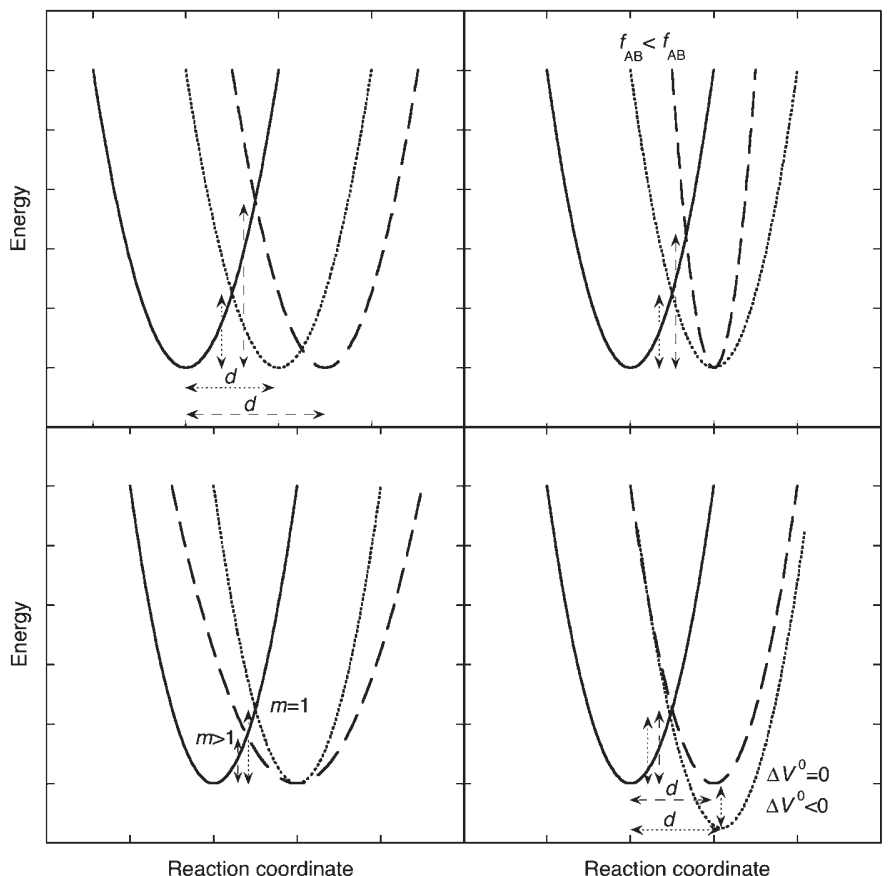

Figure 6. Illustration of how the barrier heights depend on each of the reactivity indexes of ISM. a) Sum of the bond extensions, $d=\left(l_{\mathrm{BC}}^{\neq}-l_{\mathrm{BC}, \mathrm{eq}}\right)+$ $\left(l_{\mathrm{AB}}^{ \pm}-l_{\mathrm{AB}, \mathrm{eq}}\right)$. b) Force constants, $f_{\mathrm{BC}}$ and $f_{\mathrm{AB}}$ c c) Electrophilicity index, $m$. d) Reaction energy, $\Delta V^{0}$.

The increase of the intrinsic barrier with the reaction energy, illustrated in Figure $6 \mathrm{~d}$ by a larger $d$ for the more exothermic reaction, decreases the change of the transition state structure with the change in reaction energy. For sufficiently exothermic reactions Hammond postulate will be followed and eventually the transition state structure will closely resemble the reactants structure, whereas for sufficiently endothermic reactions the transition state structure will approach that of the products. However, the increase of $\Delta G_{0}^{\neq}$with $\Delta G^{0}$ makes the Brønsted relation valid for a wider range of $\mathrm{p} K_{\mathrm{a}}$ values, and the curvature of Brønsted plots expected from Marcus equation can only be observed when very different acidities are probed. Moreover, if the curvature eventually observed is employed to calculate the intrinsic barrier, this will be overestimated.

A more serious limitation to Hammond postulate arises when the reactant and product curves are very asymmetric, as shown in Figure 6b. This is the case of reactions where a bond with much higher, or much lower, force constant is formed in the course of the reaction. For example, the $\mathrm{H}$ atom abstractions $\mathrm{CH}_{3}+\mathrm{C}_{2} \mathrm{H}_{6}$ and $\mathrm{CH}_{3} \mathrm{O}+\mathrm{C}_{2} \mathrm{H}_{6}$ are both weakly exothermic, $\Delta V^{0}=-3.8$ and $-3.1 \mathrm{kcal} \mathrm{mol}^{-1}$, but the first has an almost symmetrical transition state $\left(n^{\neq}=0.46\right)$ whereas the transition state of the second one is product- like $\left(n^{\neq}=0.22\right)$. The latter reaction is an example of a pronounced anti-Hammond behaviour that is explained by the high force constant of the $\mathrm{OH}$ bond in methanol.

Electronic effects may exert a strong influence in the barrier, as shown in Figure 6c, and under certain circumstances electronic effects may dominate over thermodynamic effects. This has been widely recognized but has been lacking a simple and quantitative expression, now found in ISM. Electronic effects associated with electrophilicity index $m$ are more accentuated when $m$ is close to unity. Typical examples are carbon acids such as nitroalkanes. The value of $m$ was estimated to decrease from $m=1.095$ for nitromethane to $m=1$ for 2-nitropropane, mostly due to the decrease in the electronic affinity originated by the electrondonating groups $\left(\sigma^{*}<0\right)$, and increase the deprotonation barrier more than the decrease in $\mathrm{p} K_{\mathrm{a}}$ can decrease that barrier. $^{[34]}$

Finally, selectivity can also be interpreted on the same grounds. In general, the free-energy dependence makes the Leffler-Hammond relationship work, and selectivity decreases with reactivity up to the limit when the most reactive reaction becomes barrierless. However, in cation-anion recombinations the nucleophilic parameter $N_{+}$proposed by Ritchie is correlated with the electrophilicity index, showing that the reactivity of these systems is controlled by electronic parameters and RSP breaks down. ${ }^{[48]}$

\section{Conclusion}

ISM unveils the role of the most important reactivity indexes that control the rates of fundamental chemical reactions, namely, atom transfers, proton transfers and $S_{N} 2$ reactions. The reactivity tends to increase as the reactive bonds become shorter, the bonds weaken, hydrogen bonding occurs, and charge shift is facilitated. The free-energy dependence of the rates is modulated by the increase of the "intrinsic" barrier with the exothermicity. This leads to a wider application of linear free-energy relationships, such as the BEP or the Brønsted relationship, in energy ranges where a quadratic (Marcus-type) dependence could already be expected.

ISM can be applied to complex systems, such as atom and proton transfers in enzymes, without any further approximations or computational labour. It is an efficient and reliable reactivity model, particularly well suited to design productive experiments. However, rather than contemplating what has been achieved with ISM, we wish to emphasise the sound theoretical basis it offers to interpret the success, and failure, of structure-reactivity relationships.

\section{Acknowledgement}

This work has been supported by the Fundação para a Ciência e Tecnologia (Portugal) and FEDER (POCI/QUI/55505/2004). 
[1] H. Eyring, J. Chem. Phys. 1935, 3, 107.

[2] J. N. Brønsted, K. L. Pederson, Z. Phys. Chem. 1924, 108, 185.

[3] J. E. Leffler, Science 1953, 117, 340.

[4] R. P. Bell, Proc. R. Soc. London 1936, A154, 414

[5] M. G. Evans, M. Polanyi, Trans. Faraday Soc. 1936, 32, 1333.

[6] G. S. Hammond, J. Am. Chem. Soc. 1955, 77, 334

[7] M. G. Evans, M. Polanyi, Trans. Faraday Soc. 1938, 34, 11.

[8] R. A. Marcus, Ann. Rev. Phys. Chem. 1964, 15, 155.

[9] R. A. Marcus, J. Phys. Chem. 1968, 72, 891.

[10] P. W. Seakins, M. J. Pilling, J. T. Niiranen, D. Gutman, L. N. Krasnoperov, J. Phys. Chem. 1992, 96, 9847.

[11] R. Atkinson, D. L. Baulch, R. A. Cox, J. N. Crowley, R. F. Hampson, R. G. Hynes, M. E. Jenkin, M. J. Rossi, J. Troe, Atoms. Chem. Phys. 2006, 6,3625 .

[12] H. Mayr, A. R. Ofial, Angew. Chem. 2006, 118, 1876; Angew. Chem. Int. Ed. 2006, 45,1844

[13] D. M. Camaioni, S. T. Autray, T. B. Salinas, J. A. Franz, J. Am. Chem. Soc. 1996, 118, 2013.

[14] L. P. Hammett, J. Am. Chem. Soc. 1937, 59, 96.

[15] R. G. Parr, R. A. Donnelly, M. Levy, W. E. Palke, J. Chem. Phys. 1978, 68, 3801.

[16] R. G. Parr, Z. Zhou, Acc. Chem. Res. 1993, 26, 256.

[17] R. G. Pearson, Acc. Chem. Res. 1993, 26, 250.

[18] R. Pearson, J. Chem. Educ. 1999, 76, 267.

[19] P. W. Ayers, R. G. Parr, J. Am. Chem. Soc. 2000, 122, 2010.

[20] A. Pross, S. Shaik, Acc. Chem. Res. 1983, 16, 363

[21] S. Shaik, P. C. Hiberty, Adv. Quantum Chem. 1995, 26, 99.

[22] S. Shaik, A. Shurki, Angew. Chem. 1999, 111, 616; Angew. Chem. Int. Ed. 1999, 38, 586.

[23] R. G. Pearson, J. Mol. Struct. 1993, 300, 519.

[24] H. S. Johnston, C. Parr, J. Am. Chem. Soc. 1963, 85, 2544.

[25] D. G. Truhlar, J. Am. Chem. Soc. 1972, 94, 7584

[26] N. Agmon, Chem. Phys. Lett. 1977, 45, 343.

[27] R. F. Nalewajski, S. J. Formosinho, A. J. C. Varandas, J. Mrozek, Int. J. Quantum Chem. 1994, 52, 1153.

[28] L. Pauling, J. Am. Chem. Soc. 1947, 69, 542.
[29] A. J. C. Varandas, F. B. Brown, C. A. Mead, D. G. Truhlar, B. C. Garrett, J. Chem. Phys. 1987, 86, 6258 .

[30] S. J. Formosinho in Theoretical and Computational Models for Organic Chemistry, Vol. C339 (Eds.: S. J. Formosinho, I. G. Csizmadia, L. G. Arnaut), NATO ASI, Kluwer, Dordrecht, 1991, pp. 159.

[31] L. G. Arnaut, S. J. Formosinho, H. D. Burrows, Chemical Kinetics, Elsevier, Amsterdam, 2007, p. 185.

[32] R. G. Parr, L. v. Szentpály, S. Liu, J. Am. Chem. Soc. 1999, 121, 1922.

[33] R. G. Pearson, Inorg. Chem. 1988, 27, 734.

[34] M. Barroso, L. G. Arnaut, S. J. Formosinho, J. Phys. Chem. A 2007, 111, 591.

[35] E. R. Lippincott, R. Schroeder, J. Chem. Phys. 1955, 23, 1099.

[36] M. Barroso, L. G. Arnaut, S. J. Formosinho, ChemPhysChem 2005, 6, 363.

[37] L. G. Arnaut, A. A. C. C. Pais, S. J. Formosinho, M. Barroso, J. Am. Chem. Soc. 2003, 125, 5236.

[38] L. G. Arnaut, S. J. Formosinho, Chem. Eur. J. 2007, 13, 8018.

[39] L. G. Arnaut, S. J. Formosinho, M. Barroso, J. Mol. Struct. 2006, 786, 207.

[40] M. Barroso, L. G. Arnaut, S. J. Formosinho, J. Phys. Org. Chem. in press.

[41] K. A. Quickert, D. J. Le Roy, J. Chem. Phys. 1970, 53, 1325.

[42] J. V. Michael, J. Chem. Phys. 1990, 92, 3394.

[43] A. J. C. Varandas, S. J. Formosinho, J. Chem. Soc. Faraday Trans. 2 1986, 953.

[44] B. G. Johnson, C. A. Gonzales, P. M. W. Gill, J. A. Pople, Chem. Phys. Lett. 1994, 221, 100.

[45] Z. Xie, J. M. Bowman, X. Zhang, J. Chem. Phys. 2006, 125, 133120.

[46] A. Dybala-Defratyka, P. Paneth, J. Pu, D. G. Truhlar, J. Phys. Chem. A 2004, 108, 2475.

[47] S. T. Banks, D. C. Clary, Phys. Chem. Chem. Phys. 2007, 9, 933.

[48] S. J. Formosinho, L. G. Arnaut, J. Mol. Struct. 1996, 371, 133.

Published online: May 19, 2008 